\title{
Autonomes Chemielernen im Fernunterricht und darüber hinaus!
}

\author{
Klemens Koch ${ }^{\star}$ and Patrik S. Locher ${ }^{\mathrm{b}}$
}

\begin{abstract}
Autonomous Distance Learning in Chemistry and Beyond! Using specific examples, some of which originate from online teaching in school during the COVID-19 lockdown in spring 2020, it is demonstrated how autonomous learning in chemistry can be supported, also in the long-term. Examples include the use of electronic media, experiments carried out at home, and discussion of current topics in the media that relate to chemistry to show how learning can be encouraged and the student motivation maintained. The aim is to encourage, on the one hand, self-guided learning to fulfill given study goals and on the other, autonomous learning out of curiosity and, later in life, to have the toolset to meet professional challenges.
\end{abstract}

Keywords: Autonomes versus selbstgesteuertes Lernen · Experimente für zuhause $\cdot$ Fernunterricht $\cdot$ Lernen aus und mit Medienberichten · Lernvideos

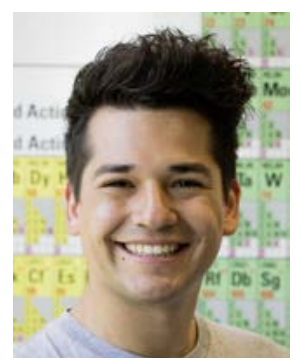

Patrik S. Locher, BSc und MSc in Chemie und molekularen Wissenschaften an der Universität Bern mit anschliessendem Erwerb des Lehrdiploms für Maturitätsschulen (PH Bern) und seit 2015 Chemielehrer an der Kantonsschule Solothurn. Mitglied des Digital Earth Centre of Excellence Switzerland, Kompetenzzentrum für digitale Lernformen, neue Lehr- und Lernformen und geographische Informationssysteme. Spezielle Interessen im Chemieunterricht: Implementierung digitaler Inhalte und Lernformen; begleitetes selbstständiges Lernen; Erklär- und Experimentiervideos.

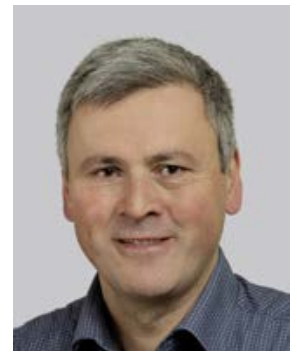

Klemens Koch, Chemiestudium an der Universität Lausanne und Dissertation in organischer Chemie sowie Ausbildung für das höhere Lehramt an der ETH Zürich. Chemielehrer seit 1995 am Gymnasium Biel-Seeland. Daneben zuerst in der Lehrerinnen- und Lehrerbildung an der ETH Zürich, heute als Fachdidaktiker Chemie an der Pädagogischen Hochschule Bern tätig. Präsident des Vereins Schweizerischer Naturwissenschaftslehrpersonen VSN (für Biologie und Chemie). Spezielle Interessen in der Chemiedidaktik: Anfangsunterricht; kleine Experimente mit grosser Lernwirkung; Elektrochemie, Thermodynamik und Quantenchemie im gymnasialen Unterricht.

\section{Einführung}

Chemielernen ist nachhaltig, wenn nach und neben der Instruktion im Unterricht und dem Chemiebuch auch andere Werkzeuge genutzt werden, um sich in chemische Fragen einzuarbeiten, und dafür auch die Motivation und Selbstwirksamkeitserwartung vorhanden ist. Mit dem World Wide Web und den vielfältigen und laufend erweiterten elektronischen Kommunikationsmöglichkeiten haben sich viele technische Freiheitsgrade eröffnet. Die im Chemieunterricht angeeigneten Kompetenzen sollen dazu dienen, auch autonom Chemie lernen zu können.
Chemielernen passiert vor allem mit Jugendlichen und jungen Erwachsenen an den Bildungsinstitutionen, den Schulen und Universitäten. Der Unterricht baut auf Vorwissen, das zuhause und im Alltag gelegt wird, bietet theoretische Modelle zur Erklärung an, vertieft, repetiert und automatisiert. Es gibt leider kaum Angebote für Erwachsene, um sich geführt oder selbstständig Grundlagen zu erarbeiten. Lebenslanges Lernen wäre erwünscht, aber wenn die Erfahrung des Lernens durch die verwendeten Lernwerkzeuge und Methoden mit den Institutionen verknüpft und an die Jugend gebunden sind, entsteht wenig Gewöhnung und Motivation für das spätere ausserinstitutionelle Lernen. Hier kann der Chemieunterricht ansetzen und Impulse geben. Ziel des Unterrichts soll auch sein, die Chemie als Wissenschaft des Alltags zu zeigen.

\subsection{Lernen in Bildungsinstitutionen}

Wir haben viel Erfahrung und Erfolg mit Lernen in Bildungsinstitutionen: Die meisten Schulen sind gut eingerichtet und die Schülerinnen und Schüler kommen mit einem klar definierten Auftrag und Pflichtenheft. Oft wird mehr Erfolg beim Erreichen der Ziele im Unterricht gewünscht, aber das Wichtigere ist ein konstruktives und offenes Lernverhalten zu fördern, das sich auch ausserhalb der Schule zeigt.

Zum Unterricht in der Schule gehören oft, und kaum hinterfragt, Hausaufgaben: Routinen trainieren, unfertige Lektionen abschliessen, Fakten durch Repetition einprägen oder Ideen nach- und vorbereiten. Dabei stellt sich die Frage, wie die spezielle Situation von Hausaufgaben genutzt werden kann, um Lernen ausserhalb und nach der Schulzeit als Selbstverständlichkeit und aus eigener Neugier zu erleben.

Eine bekannte Methode in dieser Richtung ist der Flipped Classroom, ${ }^{[1]}$ wo die Routinearbeiten des Lernens zuhause als Vorbereitung auf den Unterricht geleistet werden. Im Unterricht können die vorbereitet daherkommenden Lernenden zuerst gezielt Fragen stellen und dann mit der Lehrperson als Coach die meist anspruchsvolleren Aufgaben zum Üben, Verarbeiten und Vernetzen bearbeiten. Würden sie, wie üblich, im Unterricht das Neue lernen und dann die kognitiv anspruchsvolleren Aufgaben als Hausaufgaben lösen, wären die Lernenden mit den Herausforderungen zuhause alleine. 
Eine weitere Frage ist, was Wissen in Zeiten von Wikipedia bedeutet: Es ist sicher nicht obsolet geworden, im Gegenteil. Nur mit viel Wissen und Übung im Umgang damit, können wir die Geschehnisse der Welt passend einordnen. Trotzdem verändert der leichte Zugang zu Fakten, «alternativen Fakten» und pseudowissenschaftlichen Aussagen den Unterricht, er kann sich nicht (mehr) auf die Vermittlung von Wissen konzentrieren, sondern muss unter Ausnützung aller medialen Mittel dessen Vernetzung und Bewertung ins Zentrum stellen. Der Nutzung elektronischer Medien, der Beobachtung alltäglicher und experimenteller Phänomene und der Lektüre bzw. der Information aus Medien, ist ein neugieriger und kritischer Geist beiseitezustellen.

\subsection{Selbstgesteuertes und autonomes Lernen}

Schulen und Universitäten haben die Probleme erkannt, wenn Lernende nur dem geführten Unterricht folgen und nicht in der Lage sind, selbstorganisiert zu lernen. Viele Bildungsverantwortliche haben Anstrengungen unternommen, die Voraussetzungen für selbstorganisiertes Lernen zu verbessern, stellvertretend dafür seien hier die Unterlagen und Ergebnisse aus dem Kanton Bern angegeben. ${ }^{[2]}$ Das Problem geht aber darüber hinaus, wie Schulen für die Universitäten vorbereiten und wie dort gearbeitet werden soll. Das Ziel müsste an beiden Orten lebenslanges autonomes Lernen sein, so wie es uns aus anderen Wissensbereichen (z. B. Sprachen, Kunst, Gesellschaft, Wirtschaft, Politik) wohlbekannt ist. ${ }^{[3]}$

\section{Lernen mit elektronischen Medien}

Heute kann man davon ausgehen, dass in jedem Haushalt elektronische Geräte, seien es Smartphones, Tablets oder Laptops, zur Verfügung stehen, welche das Abrufen digitaler Inhalte über das Internet erlauben. Die Herausforderung liegt darin, aus den vielfältigen kostenlosen und -pflichtigen Angeboten im NetzDschungel die passende Auswahl zu treffen.

Beim Lernen wird ein Gefäss für die zu vermittelnden Inhalte benötigt. Bis vor einigen Jahren handelte es sich dabei meistens um gedruckte Lehrmittel in gebundener Form. Mit den heutigen technischen Möglichkeiten, wie hochauflösenden Touch-Bildschirmen und präziser Stifteingabe bei Tablets und Laptops, verändert sich auch die Art des Gefässes. Viele Verlage haben sich in den letzten Jahren am Sprung vom traditionellen Chemiebuch zum elektronischen Medium versucht. Die Interkantonale Lehrmittelzentrale beschreibt in ihrem Expertenbericht «Lehrmittel in einer digitalen Welt» vier Stufen der Digitalisierung (Abb. 1). ${ }^{[4]}$

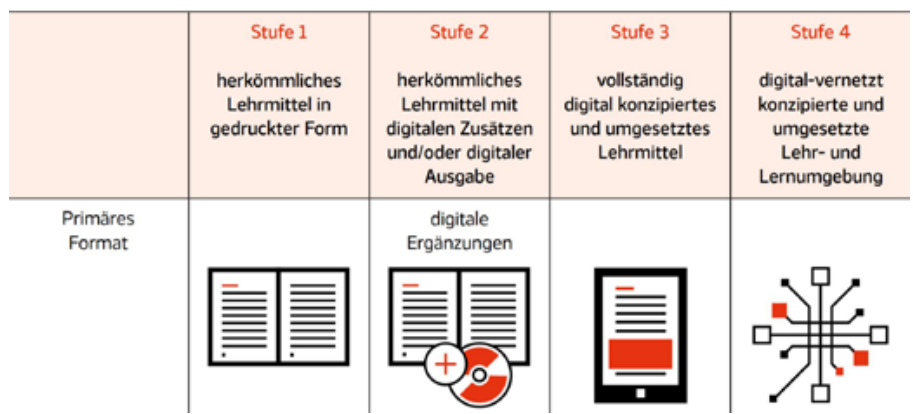

Abb. 1. Stufenmodell zu digitalen Lehrmitteln. (Expertenbericht der Interkantonalen Lehrmittelzentrale $\left.{ }^{[4]}\right)$.

Wie die Autoren schreiben, lassen sich die vier Stufen in der Realität aber nicht so klar unterscheiden. Zwei Lehrmittel der gleichen Stufe können digitale Potenziale sehr unterschiedlich nutzen. So kann ein Sachunterrichtslehrmittel viele multimediale Elemente enthalten, aber keinerlei Interaktivität zulassen, während ein Mathematiklehrmittel viele Übungen mit automatischer Korrektur, aber keine Video- oder Audioaufnahmen enthält.
Verschiedene Verlage wie Klett, Thieme, Pearson, hep und andere bieten elektronische Lehrmittel im Fach Chemie an, welche identisch mit der gedruckten Fassung des Buches sind. Über einen Zugangscode kann auf verschiedene Zusatzinhalte wie Videosequenzen, Ergänzungen, ausführliche Lösungen etc. zugegriffen werden. Solche Angebote werden der zweiten Stufe des obengenannten Modells zugeordnet.

OpensStax, ${ }^{[5]}$ eine Non-Profit Plattform für digitale Lehrbücher der Rice Universität in Huston, Texas, stellt ein englischsprachiges OER-Angebot (Open Educational Ressources) dar. Bei den Lehrbüchern handelt es sich um peer-reviewte Inhalte, welche multimedial angereichert wurden und auf interaktive Materialien verweisen. Auch Print-on-Demand Angebote sind jeweils implementiert.

Vollständig digital konzipierte Chemielehrmittel gibt es noch kaum auf dem Markt. Ein Beispiel stellt das digitale Lehrwerk Chemie von Brockhaus dar.[6] Mit Videosequenzen, digitalen Aufgaben und Übungen sowie weiterführenden Informationen bildet es ein in sich geschlossenes Angebot, welches ausschliesslich online verfügbar ist und sich auf die Nutzung im Unterricht der Sekundarstufe I ausrichtet.

Eine umfangreiche Sammlung von digitalen Lernsequenzen bietet ChemgaPedia. ${ }^{[7]}$ Kostenlos werden nach Themengebieten sortierte Lerneinheiten mit Informationen zu Schwierigkeitsgrad und geschätztem Zeitaufwand aufgelistet. Implementiert sind jeweils interaktive Elemente wie 3D-Modelle, Animationen und Übungssequenzen.

Auch alleinstehende, nicht direkt in Lehrmittel eingebundene, multimediale Inhalte sind auf verschiedenen Plattformen verfügbar. Digitale Labore für Kinder ${ }^{[8]}$ oder höhere Niveaus, ${ }^{[9]}$ Simulationen ${ }^{[10,11]}$ und interaktive Übungen ${ }^{[12,13]}$ unterstützen die Lernenden beim Vertiefen bestimmter Themen. Insbesondere für das autonome Lernen wichtig und nicht $\mathrm{zu}$ vergessen sind Plattformen, ${ }^{[14,15]}$ welche den Austausch zu bestimmten Fragen oder Themen erlauben.

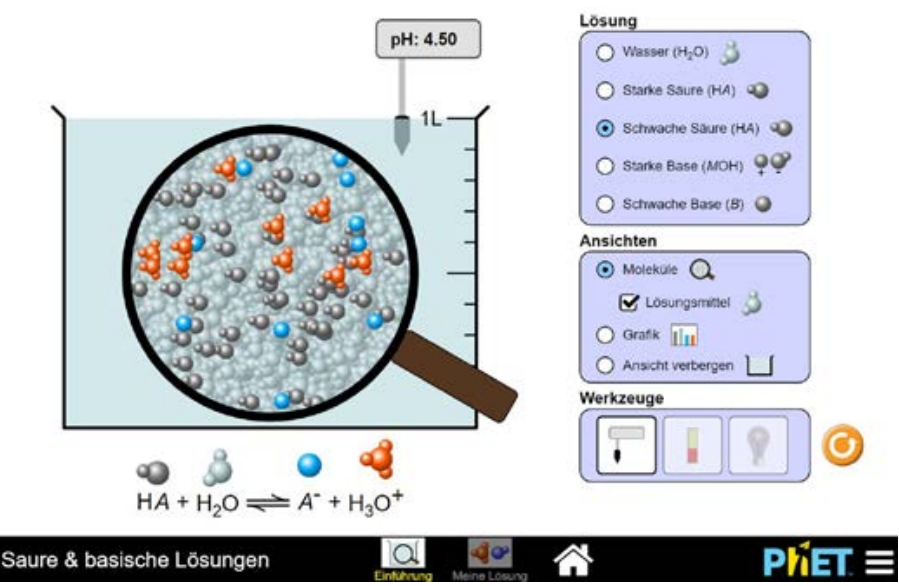

Abb. 2. Screenshot einer Simulation zu sauren und basischen Lösungen von PhET. (University of Colorado Boulder) ${ }^{[11]}$

\subsection{Lernvideos}

Audiovisuelle chemische Inhalte sind im Internet in grosser Fülle vorhanden. Von gut recherchierten und aufgearbeiteten Lernvideos über aufgenommene Vorlesungen bis hin zu kurzen Video-Snacks mit Experimenten und musikalisch vermittelten Inhalten (YouTube: acapellascience[16]) findet man auf verschiedenen Plattformen fast alles. Insbesondere auf sozialen Medien wie Instagram, Facebook und TikTok werden täglich Videos veröffentlicht, welche eindrückliche chemische Phänomene zeigen (Elefantenzahnpasta, Knallgasreaktion, Verbrennungsvorgänge 
etc.) und so einerseits die Aufmerksamkeit auf das Fach lenken, andererseits aber in den wenigsten Fällen die Möglichkeit bieten, die gezeigten Vorgänge zu analysieren und sich mit der Chemie auseinanderzusetzen (Abb. 3). Im Gegensatz dazu werden aber auch täglich Erklärvideos ins Web hochgeladen, welche echtes Lernen ermöglichen. Die Erfahrung zeigt, dass Schülerinnen und Schüler gerne Inhalte mit Lernvideos erarbeiten, sei es im Fernunterricht oder insbesondere dann, wenn der Prozess durch eine Lehrperson begleitet wird, welche situativ und individuell Unterstützung anbieten kann.

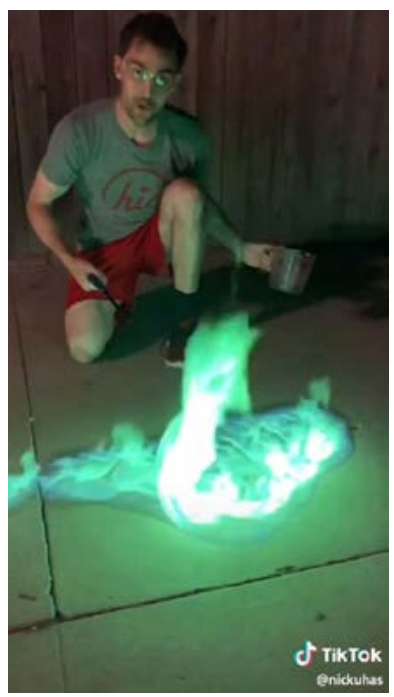

Abb. 3. Videoclip auf TikTok: Verbrennung einer Borverbindung. Der chemische Kontext wird nicht thematisiert. (TikTok/nickuhas).

Im Gegensatz zum angeleiteten Lernen besitzen Schülerinnen und Schüler beim Lernen mit Videosequenzen eine grössere Selbstbestimmung. Es ist möglich, Abschnitte zu wiederholen, die Geschwindigkeit zu verändern oder das Video zu pausieren, um Notizen zu machen. Moderne Lernvideos, wie man sie beispielsweise kostenlos auf YouTube oder weiteren Plattformen findet, sind oft ansprechend animiert und spannend anzusehen. In Kombination mit einer sympathischen Erklärstimme erhöht eine solche Produktion die Motivation der Lernenden und begünstigt den Lernerfolg. Insbesondere für das autonome Lernen stellen audiovisuelle Formate eine wichtige Ergänzung zu textbasierten Lerninhalten dar. Sie lassen sich einfach weitergeben oder speichern und ermöglichen es, zentrale Aussagen entsprechend hervorzuheben. Kanäle wie musstewissen Chemie (Abb. 4), ${ }^{[17]}$ simpleclub, ${ }^{[18]}$ Learning Level Up ${ }^{[19]}$ oder Videos der Khan Academy[20] stehen stellvertretend für zahlreiche Anbieter im

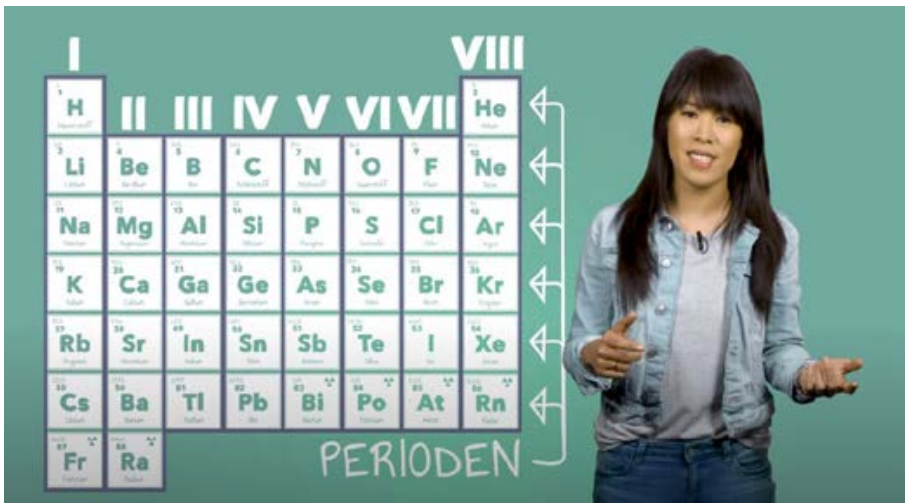

Abb. 4. Screenshot eines Erklärvideos des YouTube-Kanals musstewissen Chemie. (YouTube/musstewissen Chemie).
Bereich der lernfilmbasierten Chemievermittlung. Eine Übersicht zur Verwendung solcher Lernvideos wurde kürzlich in «Chemie \& Schule» des Verbandes der Chemielehrer/innen Österreichs veröffentlicht. [21]

Neben kostenlosen Angeboten auf Videoplattformen, wo die Qualität der Videos variiert, gibt es auch Anbieter von hochwertigen Bezahlproduktionen, beispielsweise das Institut für Film und Bild in Wissenschaft und Unterricht (FWU) ${ }^{[22]}$ oder die Gesellschaft für Information und Darstellung (GIDA). [23]

Erklärvideos bieten viele Vorteile und erhöhen die Autonomie der Lernenden. Insbesondere beim Verwenden der Sequenzen in der Schule gibt es aber auch Schwächen. So kann ein Video das Experiment in der Realität nicht ersetzen. Das Chemielernen lebt von den eigenen Erfahrungen, den Erfolgen und Misserfolgen beim praktischen Arbeiten im Labor. Schliesst man diese Erlebnisse aus, fehlt ein integraler Bestandteil der Chemie. Weiter sind die Videos in sich abgeschlossen und Änderungen können kaum angebracht werden. Allfällige fachdidaktische, sprachliche oder thematische Ergänzungen sind nicht möglich.

\subsection{Produktion eigener Lernvideos}

Insbesondere in der Zeit des Fernunterrichts haben sich viele Lehrpersonen intensiv mit Möglichkeiten beschäftigt, mit einfachen Mitteln audiovisuelle Lerninhalte anzufertigen (Abb. 5). Das Erstellen eigener Lernvideos ist mit einem mehr oder weniger grossen Aufwand verbunden. Die Anforderungen an zu verwendende Geräte und Programme orientieren sich an der Art des Videos. Geht es um einen theoretischen Aspekt, ein Experiment oder um eine Kombination? Werden die Handlungen animiert, mit Stop Motion Technik ${ }^{[24]}$ aufgenommen oder handelt es sich um eine Demonstration im Labor? Wie soll präsentiert werden? Gibt es Texteinblendungen, eine Stimme aus dem Off oder eine moderierende Person?

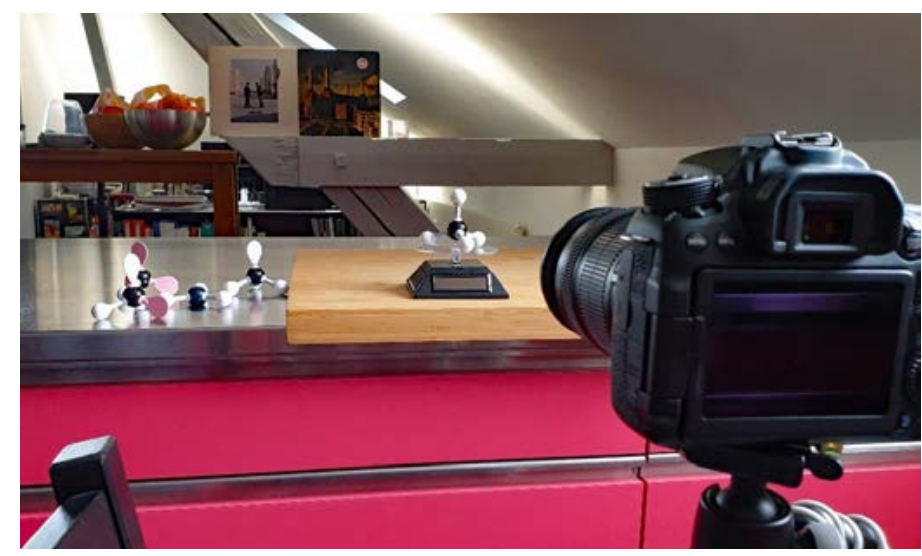

Abb. 5. Videoaufnahme zuhause beim Autor P.S.L. in der Zeit des Fernunterrichts: «Der räumliche Bau von Molekülen». (Instagram/chemie_loc).

Viele Tools, um auf pragmatische Art und Weise ein Lernvideo zu erstellen, befinden sich bereits auf dem eigenen Computer oder lassen sich mit wenig Aufwand installieren. In PowerPoint ist es möglich, einfache Animationen zu erstellen. Währenddem die PowerPoint-Präsentation auf dem Bildschirm läuft, kann der Screen mit dem Open Source Programm OBS Studio ${ }^{[25]}$ aufgenommen werden. So erhält man eine Videodatei der PowerPointPräsentation. Mit einem guten Mikrofon und einem Interface (oder alternativ einem Headset) lässt sich die Tonspur via dem Open Source Audiorekorder Audacity ${ }^{[26]}$ aufnehmen. Video- und Tonspur werden in freien Videobearbeitungsprogrammen wie beispielsweise Openshot ${ }^{[27]}$ oder Shotcut ${ }^{[28]}$ zusammengeführt, allenfalls mit Texteinblendungen versehen und anschliessend exportiert. 


\section{Autonomes Lernen mit Experimenten zuhause}

Wissen wird in der Chemie vor allem über Experimente gewonnen und das soll auch im Chemieunterricht so sein. Wo Chemie unterrichtet wird, steht fast überall eine grosse Zahl von Chemikalien, Gerätschaften und Apparaten zur Verfügung, um Demonstrationsexperimente vorzuführen oder die Lernenden experimentieren zu lassen. Doch wie geht das, wenn Lernende zuhause sind und keinen Zugang zur Infrastruktur der Bildungsinstitution und auch keine fachliche Betreuung haben? Der Lockdown im Frühling 2020 hat einfache experimentelle Ideen für zuhause gefördert, einige davon sind in Tabelle $1 \mathrm{zu}-$ sammengestellt. Weitere Hinweise auf Experimente für zuhause wurden Z. B. von der Helmholtz-Stiftung publiziert. [29]

\section{Chemie aus den Medien -}

\section{Beispiele, die lernförderliche Fragen aufwerfen}

Chemie lernen geht am besten anhand von interessanten chemischen Problemen. Meistens werden die Probleme im Unterricht von der Lehrperson gestellt. Die Klasse erarbeitet sich dann eine Lösung oder oft wird sie gleich von der Lehrperson vorgezeigt und verallgemeinert. So können weitere Probleme durch Analogie oder Deduktion bearbeitet werden, meistens in Form von Anwendungsaufgaben.

Wenn die Schülerinnen und Schüler aber aktuellen chemischen Problemen in ihrem Alltag, zum Beispiel über die Medien, begegnen, dann stellen sich die Fragen viel dringlicher und oft auch existentieller. Das soll an zwei aktuellen Beispielen mit einer

Tabelle 1. Mögliche Experimente für zuhause, ihre Durchführung und Verwendung im Chemielehrgang

\section{Experiment}

Monomolekularer Seifenfleck

Zuckerkristalle züchten

Alkoholische Gärung

Gärung im Brotteig und anschliessend Backen

Frischkäse herstellen

Butter herstellen

Butter einkochen

Essig selber herstellen und Gehalt bestimmen

Enfleurage

Limonen als Brennstoff und Lösungsmittel

\section{Zähen Kaugummi weichmachen}

(Gesättigte) Salzlösung herstellen und Untersuchungen damit

Wasser destillieren mit Sonnenenergie

\section{Durchführung}

(Sehr) wenig Seife (Messerspitze) auf eine mit Zimtpulver bestreute Wasserfläche geben.

Eine warm-gesättigte Zuckerlösung herstellen und stehen lassen.

Zuckerwasser und Hefe (in Gärgefäss) mischen. Faktoren (T, c, Hefe...) variieren und $\mathrm{CO}_{2}$ nachweisen.

Teig nach Rezept herstellen und backen.

Milch erwärmen und Casein mit Nature-Joghurt ausfällen.

Rahm schlagen, bis Butter entsteht.

Butter erhitzen, bis Wasser verdampft, Albumin denaturiert und schliesslich dehydratisiert.

Wein mit trübem Essig stehen lassen. Säuregehalt (durch Zugabe von Natron bis das Schäumen endet) abschätzen.

Aromatische Gewürze oder Orangenschale mit Stücken schwarzer Schokolade in Glas verschliessen und danach Schokolade degustieren.

Orangenschale knicken und das herausspritzende Limonen in eine Kerzenflamme oder auf einen dünnhäutigen aufgeblasenen Ballon richten.

Einen Kaugummi beim Kauen zäh werden lassen. Dann schwarze Schokolade als Weichmacher einsetzen.

Gesättigte Salzlösung aus Regeneriersalz (Vergleich mit Kochsalz?) herstellen und experimentieren.

Leitungswasser in Becken mit Plastikfolie abdecken, an Sonne stellen und Kondenswasser auffangen.

\section{Verwendung im Chemielehrgang}

Phänomen zur Teilchenstruktur der Materie und zur Untersuchung der Eigenschaften von Seife

Kristallisation, Trennungsmethoden, Struktur. Kann als Wettbewerb durchgeführt werden.

Reaktionsverlauf und seine Steuerung. Stöchiometrie, Energie in biochemischen Reaktionen

Stöchiometrie, Gemische, Reaktionen beim Backen

Eiweisse/Proteine, Denaturierung, Chemie der Küche

Gemische, Fett-in-Wasser- und Wasser-in-Fett-Emulsion

Wasser-in-Fett-Emulsion, Proteine: Denaturierung und thermische Zersetzung, Fette

Reaktionsverlauf und seine Steuerung. Stöchiometrie, Energie in biochemischen Reaktionen, Oxidation

Sublimieren/resublimieren. Aroma durch Degustation evaluieren; Objektivität, Blindprobe, Blindstudie, Doppelblindstudie bei der gewählten Methode?

Funktionelle Gruppen und Struktur des Limonen-Moleküls, Verbrennung als Übergang apolarer in polare Elektronenpaarbindungen versus «nur» zwischenmolekulare Kräfte zwischen Limonen und Gummimolekülen.

Polymere und Weichmacher, Lebensmittel und Biochemie

Dichte, Schwimmen/Sinken, Konzentration, Osmose, Verdunsten, Schichtung von Flüssigkeiten

Destillieren, Mineralsalze 
guten Einsicht in thermodynamische Fragen dargestellt werden. Beide Beispiele wurden kürzlich von den Medien aufgenommen und verbreitet.

\subsection{Wieso explodiert Ammoniumnitrat - was macht die Reaktion so spontan?}

Die tragische Explosion von ca. 2750 t Ammoniumnitrat im Hafen von Beirut am 4. August 2020 (Abb. 6) ist bereits in vielen Aspekten sehr gut aufgearbeitet, z. B. auf Wikipedia. ${ }^{[30]}$ Sie hat die Schülerinnen und Schüler verstört und zu vielen Fragen geführt. Das kann und soll der Chemieunterricht aufnehmen.

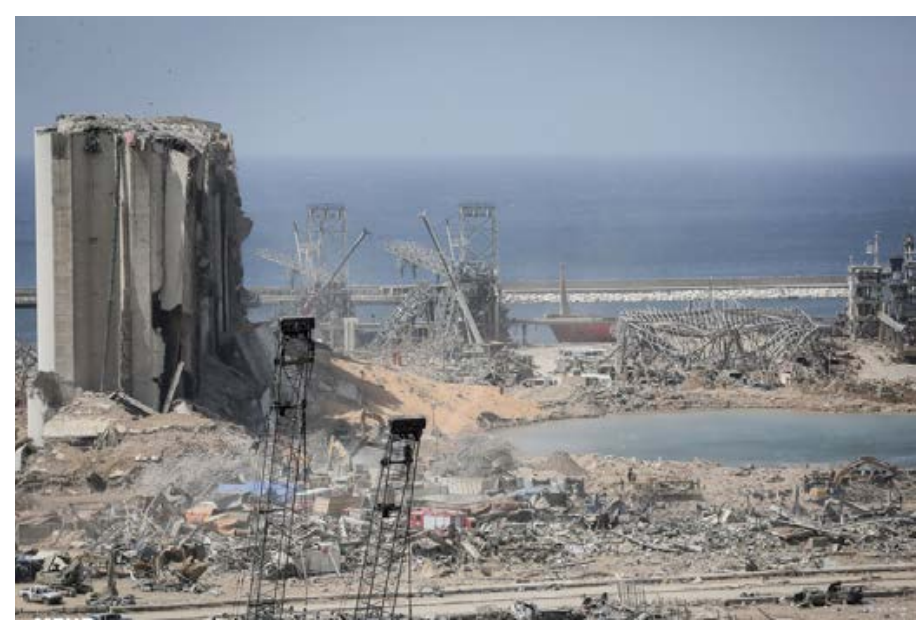

Abb. 6. Zerstörung nach der Ammoniumnitratexplosion in Beirut am 4. August 2020. https://upload. wikimedia.org/wikipedia/commons/5/5c/ Damages_after_2020_Beirut_explosions_1.jpg Licence: Mehr News Agency, CC BY $4.0<$ https://creativecommons.org/licenses/by/4.0>, via Wikimedia Commons.

Die chemischen und gesellschaftlichen Fragen aus diesem Unglück können an verschiedenen Stellen im Chemieunterricht Eingang finden: Chemie als Wissenschaft und Wirtschaftszweig; chemische Aktivitäten als Chance und Gefahr; Aggregatzustände; Stöchiometrie; Stickstoffdünger und Biochemie; Herstellung von Ammoniumnitrat; Säuren und Basen; Haber-Bosch-Prozess; Energetik, Entropie und Reaktionsantrieb; Reaktionsgeschwindigkeit; Redoxreaktionen und Oxidationszahl.

Vereinfacht kann die Explosionsreaktion wie folgt dargestellt werden.

$$
\mathrm{NH}_{4} \mathrm{NO}_{3}(\mathrm{~s}) \rightarrow 2 \mathrm{H}_{2} \mathrm{O}(\mathrm{g})+\mathrm{N}_{2}(\mathrm{~g})+1 / 2 \mathrm{O}_{2}(\mathrm{~g})
$$

Nebenreaktionen passieren sicher viele, vor allem bei den hochenergetischen Reaktionsbedingungen, wie z. B. die nitrosen Gase zeigen, welche charakteristisch sind und schon kurz nach der Explosion (Abb. 6) auf die Beteiligung von Stickstoffverbindungen und daraus folgender Entstehung von Stickstoffoxiden in der Reaktion hingewiesen haben.

Die Reaktion (1) ist thermodynamisch sehr interessant, weil ausgeprägt, ja gerade beängstigend spontan. Das zeigen qualitative Betrachtungen wie auch Berechnungen der Reaktionsenthalpie $\Delta \mathrm{H}$ $\left(\Delta \mathrm{H}^{\circ}=-118 \mathrm{~kJ} \cdot \mathrm{mol}^{-1}\right)$, der freien Reaktionsenthalpie $\Delta \mathrm{G}\left(\Delta \mathrm{G}^{\circ}=\right.$ $\left.-274 \mathrm{~kJ} \cdot \mathrm{mol}^{-1}\right)$ und der Reaktionsentropie $\Delta \mathrm{S}\left(\Delta \mathrm{S}^{\circ}=+522 \mathrm{~J} \cdot \mathrm{mol}^{-1}\right.$ $\left.\cdot \mathrm{K}^{-1}\right)$. Die schiere Wucht der Explosion, die Grösse des entstehenden Gasvolumens $\left(\mathrm{V}^{\circ} \cong 2.7\right.$ Millionen $\mathrm{m}^{3}$ aus $2750 \mathrm{t}$ Ammoniumnitrat, die Temperatur kann abgeschätzt und mit einbezogen werden) und deren offensichtlichen Bedeutungen motivieren zu stöchiometrischen Berechnungen und energetischen Betrachtungen.

\subsection{Kann Ethanol in einer spontanen Reaktion aus Kohlenstoffdioxid hergestellt werden?}

Eine andere interessante Pressemitteilung, welche in verschiedenen Medien wie 20 Minuten, ${ }^{[31]}$ der Wochenzeitung BielBienne ${ }^{[32]}$ und in seinen Pressemitteilungen publiziert worden ist, lautete, dass der Schweizer Grossverteiler Migros neuerdings aus Kohlenstoffdioxid hergestelltes Ethanol in Reinigungsmitteln verwende (Abb. 7). ${ }^{[33]}$

Das ist interessant! Sollte die folgende, stöchiometrisch vorerst einmal nur in Bezug auf die C-Atome ausgeglichene Reaktionsgleichung so einfach ablaufen, dann wäre das Klimaproblem gelöst und ein breit einsetzbarer Energieträger könnte auf einfachem Weg hergestellt werden. Auch die Produktion von Alkohol für die Party oder Brennsprit für das Fonduerechaud wäre denkbar.

$$
2 \mathrm{CO}_{2}(\mathrm{~g})+\ldots \rightarrow \mathrm{CH}_{3} \mathrm{CH}_{2} \mathrm{OH}(\mathrm{l})+\ldots
$$

Diese Reaktion kann mit der in umgekehrter Richtung ablaufenden, freiwilligen und gut bekannten Verbrennungsreaktion von Ethanol (3) mit $\Delta \mathrm{H}^{\circ}=-1234 \mathrm{~kJ} \cdot \mathrm{mol}^{-1}, \Delta \mathrm{G}^{\circ}=-1300 \mathrm{~kJ} \cdot \mathrm{mol}^{-1}$ und $\Delta \mathrm{S}^{\circ}=+219 \mathrm{~J} \cdot \mathrm{mol}^{-1} \cdot \mathrm{K}^{-1}$ verglichen werden:

$$
\mathrm{CH}_{3} \mathrm{CH}_{2} \mathrm{OH}(\mathrm{l})+3 \mathrm{O}_{2}(\mathrm{~g}) \rightarrow 2 \mathrm{CO}_{2}(\mathrm{~g})+3 \mathrm{H}_{2} \mathrm{O}(\mathrm{g})
$$

Können wir einfach die Umkehrung schreiben und auf dem Papier Ethanol aus Kohlenstoffdioxid entstehen lassen?

$$
2 \mathrm{CO}_{2}(\mathrm{~g})+3 \mathrm{H}_{2} \mathrm{O}(\mathrm{g}) \rightarrow \mathrm{CH}_{3} \mathrm{CH}_{2} \mathrm{OH}(\mathrm{l})+3 \mathrm{O}_{2}(\mathrm{~g}) \quad(4)=-(3)
$$

Das war auch den Schülerinnen und Schülern suspekt: Wenn die Verbrennung (3) so gut abläuft, kann die Umkehrreaktion (4) doch nicht ebenso spontan ablaufen, die thermodynamischen Änderungen hätten umgekehrte Vorzeichen wie bei (3), also wären bei (4): $\Delta \mathrm{H}^{\circ}=+1234 \mathrm{~kJ} \cdot \mathrm{mol}^{-1}, \Delta \mathrm{G}^{\circ}=+1300 \mathrm{~kJ} \cdot-$ $\mathrm{mol}^{-1}$ und $\Delta \mathrm{S}^{\circ}=-219 \mathrm{~J} \cdot \mathrm{mol}^{-1} \cdot \mathrm{K}^{-1}$. Wären beide spontan, würden die Reaktionen (3) und (4) dauernd freiwillig im Kreis laufen und dabei noch Energie abgeben! Das spricht gegen die wissenschaftlich geschulte Intuition von Experten und Lernenden. Das Dilemma in Fachbegriffen: Ein Perpetuum mobile kann nicht existieren, egal ob es Energie «aus Nichts» erzeugt, also den ersten Hauptsatz der Thermodynamik verletzt oder Energie einfach aus einem Wärmereservoir zieht, ohne Wärmefluss zwischen zwei Reservoiren unterschiedlicher Temperatur, und so «nur» den zweiten Hauptsatz der Thermodynamik verletzt.

So fragen wir uns, wie denn genau dieser Alkohol hergestellt werden könnte, wenn der naheliegende Weg nicht begangen werden kann und auch, wieso ein offensichtlich falscher Reaktionsweg als klimaschonend angepriesen wird. Die Sache ist wie bei vielen Diskussionen im Bereich Nachhaltige Entwicklung erstens komplexer als erwartet und zweitens bei genauer Hinsicht eben doch klimaschonend, wie der Artikel korrekt, allerdings mit (zu?) stark vereinfachten Argumenten, behauptet. $\mathrm{Ob}$ eine vereinfachte oder sogar widersprüchliche Erklärung in einer Publikumsreportage zulässig ist, kann mit Schülerinnen und Schüler sehr kontrovers diskutiert werden. Wenn dahinter eine mangelnde naturwissenschaftliche Bildung oder eine mangelnde Bereitschaft, sich auf differenzierte Fragen einzulassen vermutet wird, müssen wir uns fragen: Wie können Grundlagen für aktuell drängende Probleme so vernachlässigt werden und in den Redaktionen auf keine Beachtung und kritisch-informierte Auseinandersetzung treffen?

Doch wie könnte Ethanol aus Kohlenstoffdioxid entstehen, wenn nicht aus der Umkehrung der Verbrennungsreaktion? Zwei 
Möglichkeiten, Ethanol mit den beiden folgenden Reaktionen aus Kohlenstoffdioxid herzustellen, können leicht selber gefunden und beurteilt werden:

$$
2 \mathrm{CO}_{2}(\mathrm{~g})+3 \mathrm{H}_{2}(\mathrm{~g}) \rightarrow \mathrm{CH}_{3} \mathrm{CH}_{2} \mathrm{OH}(\mathrm{l})+1.5 \mathrm{O}_{2}(\mathrm{~g})
$$

$$
2 \mathrm{CO}_{2}(\mathrm{~g})+6 \mathrm{H}_{2}(\mathrm{~g}) \rightarrow \mathrm{CH}_{3} \mathrm{CH}_{2} \mathrm{OH}(\mathrm{l})+3 \mathrm{H}_{2} \mathrm{O}(\mathrm{l})
$$

Reaktion (5) produziert elementaren Sauerstoff, währenddem Reaktion (6) zum stabileren Produkt Wasser führt, was die Reaktion besser antreiben sollte. Eine Berechnung mit Tabellenwerten zeigt, dass die erste Reaktion (5) noch endergonisch $\left(\Delta \mathrm{H}^{\circ}=+508 \mathrm{~kJ} \cdot \mathrm{mol}^{-1}, \Delta \mathrm{G}^{\circ}=+613 \mathrm{~kJ} \cdot \mathrm{mol}^{-1}, \Delta \mathrm{S}^{\circ}=-353\right.$ $\left.\mathrm{J} \cdot \mathrm{mol}^{-1} \cdot \mathrm{K}^{-1}\right)$, die zweite (6) aber bereits exergonisch, also spontan, verläuft $\left(\Delta \mathrm{H}^{\circ}=-350 \mathrm{~kJ} \cdot \mathrm{mol}^{-1}, \Delta \mathrm{G}^{\circ}=-98 \mathrm{~kJ} \cdot \mathrm{mol}^{-1}, \Delta \mathrm{S}^{\circ}=-843\right.$ $\left.\mathrm{J} \cdot \mathrm{mol}^{-1} \cdot \mathrm{K}^{-1}\right)$.

Eine Möglichkeit wäre, nicht Kohlenstoffdioxid einzusetzen, sondern energiereicheres Kohlenstoffmonoxid. Damit liesse sich Ethanol energetisch weniger aufwändig erstellen.

$$
2 \mathrm{CO}(\mathrm{g})+3 \mathrm{H}_{2}(\mathrm{~g}) \rightarrow \mathrm{CH}_{3} \mathrm{CH}_{2} \mathrm{OH}(\mathrm{l})+0.5 \mathrm{O}_{2}(\mathrm{~g}) \text { bzw. }
$$

$$
2 \mathrm{CO}(\mathrm{g})+4 \mathrm{H}_{2}(\mathrm{~g}) \rightarrow \mathrm{CH}_{3} \mathrm{CH}_{2} \mathrm{OH}(\mathrm{l})+1 \mathrm{H}_{2} \mathrm{O}(\mathrm{l})
$$

Beide Reaktionen zeigen bei den Berechnungen mit Tabellenwerten tatsächlich tiefere $\Delta \mathrm{G}$-Werte, bei Reaktion (7) noch positiv $\left(\Delta \mathrm{H}^{\circ}=-56 \mathrm{~kJ} \cdot \mathrm{mol}^{-1}, \Delta \mathrm{G}^{\circ}=+99 \mathrm{~kJ} \cdot \mathrm{mol}^{-1}, \Delta \mathrm{S}^{\circ}=\right.$ $-523 \mathrm{~J} \cdot \mathrm{mol}^{-1} \cdot \mathrm{K}^{-1}$ ), aber bei Reaktion (8) ist die Differenz $\Delta \mathrm{G}$ schon negativ, die Reaktion also spontan $\left(\Delta \mathrm{H}^{\circ}=-342 \mathrm{~kJ} \cdot \mathrm{mol}^{-1}\right.$, $\left.\Delta \mathrm{G}^{\circ}=-138 \mathrm{~kJ} \cdot \mathrm{mol}^{-1}, \Delta \mathrm{S}^{\circ}=-689 \mathrm{~J} \cdot \mathrm{mol}^{-1} \cdot \mathrm{K}^{-1}\right)$. Ein Blick auf die Webseite der Firma Lanzatech, ${ }^{[34]}$ welche das klimafreundliche Ethanol herstellt, zeigt, dass tatsächlich Kohlenstoffmonoxid aus den (stark komprimierten) Abgasen von Stahlwerken in China verwendet wird, dagegen zeigt das «Informationsvideo» der Migros ${ }^{[34]}$ und der Pendlerzeitung «20 Minuten» eindeutig Kohlenstoffdioxidmoleküle (Abb. 7a). ${ }^{[33]}$

a)

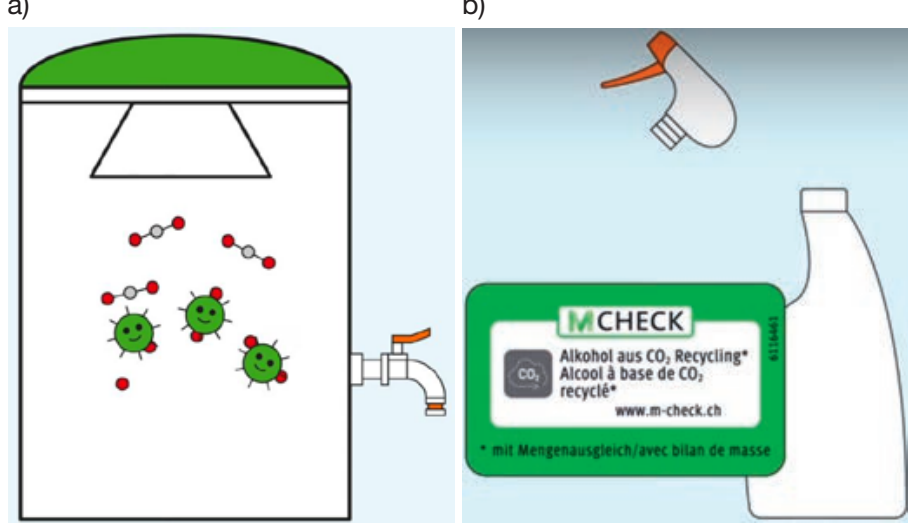

Abb. 7. a) Grafik zur Herstellung des Alkohols in einem Gärtank für die Reinigungsmittel. Screenshot aus einem Informationsvideo der Migros mit der Erklärung: «Wir fermentieren $\mathrm{CO}_{2}$ mithilfe von Mikroorganismen. Bei der Gärung entsteht dann der Alkohol». b) Angaben zum Inhalt des Reinigungsmittels auf der Etikette (Migros ${ }^{[33]}$ ).

Der chemische Hintergrund des klimafreundlichen Prozesses wurde durch einen längeren Mailwechsel mit dem Grossverteiler

Migros, der Ökobilanzerstellerin Carbotech, dem das Projekt unterstützendenden WWF und mit ihren hilfreichen, offenen und umfassenden Antworten klar: Die Lösung liegt sowohl im Einsatz von Kohlenstoffmonoxid wie auch von Wasserstoff.

Grundlage ist eine, auf den ersten Blick erstaunliche, Reaktion, welche das 1994 erstmals in Kaninchenkot gefundene Bakterium Clostridium autoethanogenum beherrscht:[35,36] Die WassergasShift-Reaktion ${ }^{[37]}$ (9) ist aus der technischen Chemie bekannt, um Kohlenstoffmonoxid aus Abgasen zu entfernen und Wasserstoff zu produzieren. Die Reaktion (9) ist nur leicht exergonisch $\left(\Delta \mathrm{H}^{\circ}\right.$ $\left.=+4 \mathrm{~kJ} \cdot \mathrm{mol}^{-1}, \Delta \mathrm{G}^{\circ}=-20 \mathrm{~kJ} \cdot \mathrm{mol}^{-1}, \Delta \mathrm{S}^{\circ}=+77 \mathrm{~J} \cdot \mathrm{mol}^{-1} \cdot \mathrm{K}^{-1}\right)$ und bietet sich an, um geeignete Reaktionsbedingungen, z. B. hoher Druck, für ihren Ablauf zu diskutieren.

$$
\mathrm{CO}(\mathrm{g})+\mathrm{H}_{2} \mathrm{O}(\mathrm{l}) \rightarrow \mathrm{H}_{2}(\mathrm{~g})+\mathrm{CO}_{2}(\mathrm{~g})
$$

Mit dem in Reaktion (9) erzeugten Wasserstoff kann weiteres Kohlenstoffmonoxid reduziert werden und kombiniert mit Reaktion (8) kann die Gesamtreaktion (10) $\left(\Delta \mathrm{H}^{\circ}=-326 \mathrm{~kJ} \cdot \mathrm{mol}^{-1}\right.$, $\left.\Delta \mathrm{G}^{\circ}=-218 \mathrm{~kJ} \cdot \mathrm{mol}^{-1}, \Delta \mathrm{S}^{\circ}=-381 \mathrm{~J} \cdot \mathrm{mol}^{-1} \cdot \mathrm{K}^{-1}\right)$ zur Herstellung von Ethanol $\mathrm{CH}_{3} \mathrm{CH}_{2} \mathrm{OH}$ (1) formuliert werden:

$$
\begin{aligned}
& 4 \mathrm{CO}(\mathrm{g})+4 \mathrm{H}_{2} \mathrm{O}(\mathrm{l}) \rightarrow \\
& 4 \mathrm{H}_{2}(\mathrm{~g})+4 \mathrm{CO}_{2}(\mathrm{~g}) \\
& 2 \mathrm{CO}(\mathrm{g})+4 \mathrm{H}_{2}(\mathrm{~g}) \rightarrow \\
& \mathrm{CH}_{3} \mathrm{CH}_{2} \mathrm{OH}(\mathrm{l})+1 \mathrm{H}_{2} \mathrm{O}(\mathrm{l})
\end{aligned}
$$$$
6 \mathrm{CO}(\mathrm{g})+3 \mathrm{H}_{2} \mathrm{O}(\mathrm{l}) \rightarrow
$$$$
\mathrm{CH}_{3} \mathrm{CH}_{2} \mathrm{OH}(\mathrm{l})+4 \mathrm{CO}_{2}(\mathrm{~g})
$$

Alle Reaktionen und Varianten können thermodynamisch mit Tabellenwerten im Hinblick auf Spontaneität und Wärmeabgabe berechnet werden und bei diesem Beispiel ist die Motivation der Lernenden gross, weil der Sinn leicht erkannt wird und nachhaltige Prozesse, wie auch die Aufklärung der Widersprüche in der Kommunikation, als spannend und hoch bedeutend beurteilt werden.

Fazit: Damit sind wir bei einer Gesamtreaktion (10), welche tatsächlich Ethanol produziert, wenn auch aus Kohlenstoffmonoxid statt Kohlenstoffdioxid. Es entsteht sogar zusätzlich Kohlenstoffdioxid! Der Prozess ist trotzdem klimafreundlich, weil das Kohlenstoffmonoxid sonst abgefackelt würde und die alternative Verwendung den Kohlenstoffdioxidfluss in die Atmosphäre vermindert. In der Kommunikation der Branche wird dabei von $\ll \mathrm{CO}_{2}$ - ̈̈quivalenten nach IPCC $2013{ }^{[38]}$ gesprochen. Dass damit aber immer noch nicht Ethanol aus Kohlenstoffdioxid hergestellt wird, ja sogar zusätzliches Kohlenstoffdioxid in die Atmosphäre gerät, sei ein häufiger «Trade-off» in der Kommunikation mit dem Publikum. Wenn Wasserstoffquellen zur Verfügung stehen, kann auch direkt aus Kohlenstoffdioxid Ethanol entstehen. Das verbessert aber die Gesamtbilanz nicht, wenn man den Aufwand für die Wasserstoffgewinnung bedenkt.

Den Lernenden sind Vertrauen, Ehrlichkeit und die Möglichkeit, sich ökologisch zu verhalten, sehr wichtig. Offene Entscheidungssituationen in Fragen der nachhaltigen Entwicklung oder Ungenauigkeiten, Vereinfachungen oder sogar Fehler in der Kommunikation können sie zuerst enttäuschen. Die zu Beginn geführte und später autonome Arbeit mit Wissen in komplexen Fragestellungen macht Hintergründe besser verständlich, Argumentationen differenzierter und Beurteilungen sorgfältiger abgewogen.

\section{Schlussbemerkungen}

Wissen ist heute über (elektronische) Medien technisch so leicht zugänglich wie noch nie. Unser Alltag und die zunehmende Nutzung der wissenschaftlichen Fortschritte erfordern immer mehr davon. Wissen muss aber persönlich angeeignet und kritisch 
beurteilt werden, das kann im Chemieunterricht aber auch zuhause mit Erfahrungen und selber durchgeführten Experimenten geübt werden. Das ist eine gute Voraussetzung, um sich auch künftig auf uns zukommende Fragen mit Neugier und Vertrauen in die eigene Fähigkeit zu stellen, sie zu verstehen, am Schluss zu beurteilen und Entscheidungen verantwortungsbewusst mitzugestalten und mitzutragen. Damit kann der Chemieunterricht einen wichtigen Beitrag zur «erweiterten Gesellschaftsreife» von Lernenden leisten, die zum Beispiel in Artikel 5 der MaturitätsAnerkennungsverordnung[39] gefordert wird.

\section{Verdankungen}

Herzlichen Dank den Kollegien an der Kantonsschule Solothurn und am Gymnasium Biel für die inspirierenden Diskussionen, den Schülerinnen, Schülern und Studierenden in Solothurn, Biel und an der PH Bern für die Mitarbeit bei den Praxiserprobungen sowie dem Pressedienst der Migros und weiterer Organisationen für die hilfreiche Korrespondenz. hältlich.

Weitere Materialien zum Unterricht sind bei den beiden Autoren er-

Eingegangen: 15. November, 2020

[1] L. Abeysekera, P. Dawson, High. Edu. Res. Devel. 2015, 34, 1, https://doi.org/10.1080/07294360.2014.934336.

[2] R. Hilbe, W. Herzog, 'Selbst organisiertes Lernen am Gymnasium Theoretische Konzepte und empirische Erkenntnisse', Mittelschul- und Berufsbildungsamt, Erziehungsdirektion des Kantons Bern, 2016, Der Bericht ist als Download erhältlich: www.erz.be.ch/sol.

[3] F. E. Weinert, Unterrichtswissenschaft 1982, 10, 99.

[4] B. Döbeli Honegger, M. Hielscher, W. Hartmann, 'Lehrmittel in einer digitalen Welt', Expertenbericht im Auftrag der Interkantonalen Lehrmittelzentrale (ilz). 2018, Online verfügbar unter www.ilz.ch https://www.ilz.ch/download/374 (Zugriff 30.10.2020)

[5] https://openstax.org/details/books/chemistry-2e (Zugriff 30.10.2020)

[6] https://brockhaus.de/info/schulen/digitale-lehrwerke/chemie-7-8/ (Zugriff 30.10.2020)

[7] http://www.chemgapedia.de/vsengine/topics/de/Chemie/index.html (Zugriff 30.10.2020)

[8] https://basf.kids-interactive.de/ (Zugriff 30.10.2020)

[9] http://chemcollective.org/ (Zugriff 30.10.2020)

[10] http://mw.concord.org/nextgen/\#interactives/chemistry/ (Zugriff 30.10.2020)

[11] https://phet.colorado.edu/en/simulations/filter?subjects=chemistry\&sort=alpha\&view=grid (Zugriff 30.10 .2020$)$

[12] https://learningapps.org/index.php?category=12\&s= (Zugriff 30.10.2020)

[13] https://h5p.org/ (Zugriff 05.11.2020)

[14] https://www.reddit.com/r/chemhelp/ (Zugriff 30.10.2020)

[15] http://www.chemikerboard.de/ (Zugriff 30.10.2020)

[16] https://www.youtube.com/channel/UCTev4RNBiu6lqtx8z1e87fQ (Zugriff 12.11.2020)
[17] https://www.youtube.com/channel/UC146qqkUMTrn4nfSSOTNwiA (Zugriff 30.10.2020)

[18] https://www.youtube.com/user/TheSimpleChemics (Zugriff 30.10.2020)

[19] https://www.youtube.com/c/LearningLevelUp/ (Zugriff 30.10.2020)

[20] https://de.khanacademy.org/ (Zugriff 30.10.2020)

[21] M. Knapp, S. P. Harmer, K. Groß, Chemie \& Schule 2020, 35, 5

[22] https://www.fwu-mediathek.de/ (Zugriff 30.10.2020)

[23] https://www.gida.de/chemie (Zugriff 30.10.2020)

[24] N. Ulrich, Unterricht Chemie 2020, 177/178, 38

[25] https://obsproject.com/de (Zugriff 30.10.2020)

[26] https://www.audacityteam.org/ (Zugriff 30.10.2020)

[27] https://www.openshot.org/de/ (Zugriff 30.10.2020)

[28] https://shotcut.org/ (Zugriff 30.10.2020)

[29] https://www.helmholtz.de/forschung/aktuelles/experimente-fuer-zuhause/ (Zugriff 14.10.2020)

[30] https://de.wikipedia.org/wiki/Explosionskatastrophe_in_Beirut_2020 (Zugriff 17. 10. 2020)

[31] https://www.20min.ch/video/migros-recycelt-co2-fuer-reinigungsmittel -488185968955 (Zugriff 19. 10. 2020)

[32] Redaktion des Biel-Bienne bb: 'Durch das Recyceln des $\mathrm{CO}_{2}$ (...) spart die Migros im Bereich Wasch- und Reinigungsmittel herkömmlich produzierten Alkohol ein (...)', S. 15, Nr. 37, Ausgabe vom 9./10. September 2020 der Wochenregionalzeitung Biel-Bienne) zum Download auf https://www.bielbienne.com/de/bielbienne-archiv.html (Zugriff 19. 10. 2020)

[33] Pressedienst Migros: 'Migros recycelt $\mathrm{CO}$ für Reinigungsmittel', 24.08.2020, https://generation-m.migros.ch/de/nachhaltige-migros/co2-recycling.html und Video darauf (Zugriff 19. 10. 2020)

[34] Video auf https://www.lanzatech.com (Zugriff 21.09.2020)

[35] https://en.wikipedia.org/wiki/Clostridium_autoethanogenum (Zugriff 27.10.2020)

[36] J. Abrini, H. Naveau, E. Nyns, Arch. Microbiol. 1994, 161, 345 https://doi.org/10.1007/BF00303591

[37] https://de.wikipedia.org/wiki/Wassergas-Shift-Reaktion (Zugriff 27.10.2020)

[38] IPCC, 'Summary for Policymakers', in 'Climate Change 2013: The Physical Science Basis.Contribution of Working Group I to the Fifth Assessment Report of the Intergovernmental Panel on Climate Change', 2013, Cambridge University Press, Cambridge, United Kingdom and New York, NY, USA, https://www.ipcc.ch/site/assets/uploads/2018/02/ WG1AR5_SPM_FINAL.pdf

[39] 'Verordnung über die Anerkennung von gymnasialen Maturitätsausweisen', (Maturitäts-Anerkennungsverordnung, MAV) vom 15. Februar 1995 https://www.admin.ch/opc/de/classified-compilation/19950018/index. html

\section{License and Terms}



This is an Open Access article under the terms of the Creative Commons Attribution License CC BY 4.0. The material may not be used for commercial purposes.

The license is subject to the CHIMIA terms and conditions: (http:// chimia.ch/component/sppagebuilder/?view = page\&id=12).

The definitive version of this article is the electronic one that can be found at https://doi.org/10.2533/chimia.2021.67 